\title{
Rubrication of text information based on the voting of intellectual classifiers
}

\author{
M. Dli ${ }^{1}$, O. Bulygina ${ }^{1 *}$, A. Sokolov ${ }^{2}$ \\ 1 The Branch of National Research University MPEI in Smolensk, Russia \\ ${ }^{2}$ National Research University MPEl, Russia \\ *baguzova_ov@mail.ru
}

\begin{abstract}
The practical implementation of the concept of electronic government is one of the priorities of Russian state policy. The organization of effective interaction between authorities and citizens is an important element of this concept. In addition to providing public services, it should include the processing of electronic appeals (applications, complaints, suggestions, etc.). Research has shown that the speed and efficiency of appeal processing largely depend on the quality of determining the thematic rubric, i.e. solving the rubrication task. The analysis of citizens' appeals received by the e-mail and official websites of public authorities has revealed several specific features (small size, errors in the text, free presentation style, description of several problems) that do not allow the successful application of traditional approaches to their rubrication. To solve this problem, it has been proposed to use various methods of intellectual analysis of unstructured text data (in particular, fuzzy logical algorithms, fuzzy decision trees, fuzzy pyramidal networks, neuro-fuzzy classifiers, convolutional and recurrent neural networks). The article describes the conditions of the applicability of six intellectual classifiers proposed for rubricating the electronic citizens' appeals. They are based on such factors as the size of the document, the degree of intersection of thematic rubrics, the dynamics of their thesauruses, and the amount of accumulated statistical information. For a situation where a specific model cannot make an unambiguous choice of a thematic rubric, it is proposed to use the classifier voting method, which can significantly reduce the probability of rubrication errors based on the weighted aggregation of solutions obtained by several models selected using fuzzy inference.
\end{abstract}

Keywords: electronic citizens' appeals, rubrication of text documents, intellectual classifiers, classifier voting

For citation: Dli M., Bulygina 0., Sokolov A. Rubrication of text information based on the voting of intellectual classifiers. Prikladnaya informatika $=$ Journal of Applied Informatics, 2020, vol. 15, no. 5, pp. 29-36. DOI: 10.37791/2687-0649-2020-15-5-29-36 